\title{
Implantação do sistema eletrônico de informações em uma Universidade Pública Federal: percepção dos servidores da área de gestão de pessoas
}

A gestão de processos é de grande relevância e traz vários benefícios para todas as organizações, portanto, também vem sendo utilizada pelas instituições públicas para a melhoria dos seus serviços. Considerando que os atores envolvidos nestes processos têm papel fundamental para o sucesso que se espera de uma ferramenta de gestão, o presente artigo teve como objetivo analisar a percepção dos servidores da área de Gestão de Pessoas da Universidade Tecnológica Federal do Paraná (UTFPR) quanto à implantação de um novo sistema de gestão de processos, o SEI - Sistema Eletrônico de Informações. Para tanto, foi elaborado um questionário no modelo estruturado, composto por questões sociodemográficas, funcionais, e questões relacionadas ao sistema estudado, abordando temas como a capacitação para o seu uso, frequência de utilização, e quesitos para medir a percepção dos usuários quanto à nova ferramenta de trabalho, sendo aplicado na Reitoria e também nos 13 câmpus da UTFPR. Após a análise dos dados obtidos, constatou-se que apesar de ter representado significativas mudanças nas rotinas de trabalho dos setores investigados, a maioria dos servidores da área de gestão de pessoas aprova e apoia a utilização do Sistema Eletrônico de Informações, e que a sua implantação foi, portanto, benéfica para esta área.

\section{Implementation of the electronic information system at a Federal Public University: perception of employees of people management area}

\begin{abstract}
Process management is of great relevance and it brings several benefits to all organizations, therefore, it has also been used by public institutions to improve their services. Considering that the actors involved in these processes play a fundamental role for the success that is expected from a management tool, this article is aimed to analyze the perception of the employees of the People Management area of Federal Technological University of Paraná (UTFPR) regarding the implementation of a new process management system, the Electronic Information System - SEl. For that, a questionnaire was applied in the main and also in the 13 campuses. It was elaborated in the structured model, composed of sociodemographic, functional questions, and issues related to the studied system, addressing topics such as training for its use, frequency of use, and questions to measure users' perception of the new work tool. After analyzing the collected data, it was found that despite having represented significant changes in the work routines of the investigated departments, the majority of employees in the People Management area approve and support the use of the Electronic Information System, and that its implementation was, therefore, beneficial for this area.
\end{abstract}

Keywords: Process Management; People Management; Electronic Information System; Work Routine.

Topic: Gestão Pública

Reviewed anonymously in the process of blind peer.

Laís de Andrade Farias (iD)

Universidade Tecnológica Federal do Paraná, Brasil http://lattes.cnpq.br/3548590896553828

http://orcid.org/0000-0001-9011-5095

laisfarias@utfpr.edu.br

Letícia Calsavara de Oliveira (iD)

Universidade Tecnológica Federal do Paraná, Brasil

http://lattes.cnpq.br/9035574736027147

http://orcid.org/0000-0001-6238-0991

Icoliveira@utfpr.edu.br

Jair de Oliveira (it

Universidade Tecnológica Federal do Paraná, Brasil

http://lattes.cnpq.br/3690696890351902

http://orcid.org/0000-0001-7847-698X

jair@utfpr.edu.br

DOI: 10.6008/CBPC2179-684X.2020.004.0015
Received: 07/10/2020

Approved: 21/12/2020

\author{
Ariel Orlei Michaloski (iD) \\ Universidade Tecnológica Federal do Paraná, Brasil \\ http://lattes.cnpq.br/3016288410389201 \\ http://orcid.org/0000-0001-5802-3764 \\ ariel@utfpr.edu.br
}

Referencing this:

FARIAS, L. A.; OLIVEIRA, L. C.; OLIVEIRA, J.; MICHALOSKI, A. O.. Implantação do sistema eletrônico de informações em uma Universidade Pública Federal: percepção dos servidores da área de gestão de pessoas. Revista Brasileira de Administração Científica, v.11, n.4, p.189-199, 2020. DOI: http://doi.org/10.6008/CBPC2179$\underline{684 X .2020 .004 .0015}$ 


\section{INTRODUÇÃO}

Visando a melhoria da gestão dos órgãos do governo, tem-se observado no Brasil, nos últimos anos, o crescimento da utilização de projetos e iniciativas de gestão pública que envolvem o uso de Tecnologias da Informação e Comunicação. Em alguns casos, a simples disponibilização de informações e o acesso digital vêm provocando uma nova maneira de o cidadão relacionar-se com o governo, gerando, assim, formas inovadoras de administrar a máquina pública (ARAÚJO, 2018).

Além dessa convergência informacional, que pode ser observada nas mais diversas instituições do setor público, a utilização de ferramentas de gestão também ganhou mais popularidade. De acordo com Kezner (2013, citado por SILVA et al., 2019), as organizações passaram a utilizar os princípios da gestão de projetos, reconhecendo que seu uso poderia auxiliar na efetivação de planos estratégicos e operacionais.

Nesse aspecto, considerando a questão operacional do serviço público, cabe destacar que todos os órgãos têm a necessidade de gerenciar seus servidores, executando todas as atividades relacionadas às suas vidas funcionais. Nas autarquias, os processos relacionados à gestão de pessoas dos servidores são de responsabilidade da área de Recursos Humanos, e a burocracia presente nas regras e procedimentos dessa área, muitas vezes aplicada de maneira desatualizada, tem causado alguns problemas que prejudicam o andamento eficiente, prático e objetivo de tais processos (PEREIRA et al., 2016).

Tratando-se de recursos humanos, Domingues et al. (2015) defendem que as pessoas são o centro dos processos, e que, em uma organização, são elas que executam as atividades e implementam possíveis melhorias, elas são a base para o seu funcionamento e para o aperfeiçoamento de seus processos. Tal condição tem tanta relevância, que uma das vertentes da gestão por processos é justamente o foco nas pessoas.

Neste sentido, considerar a opinião das pessoas que estão diretamente envolvidas nos processos, frente à implantação de um novo sistema de gestão de processos, é de total relevância para que a melhoria que se busca seja efetiva. Assim, o presente artigo objetiva identificar a percepção dos servidores da área de Gestão de Pessoas da Universidade Tecnológica Federal do Paraná quanto à implantação do Sistema Eletrônico de Informações, bem como se essa implantação trouxe melhorias para as rotinas de trabalho dessa área.

Para tanto, foi realizada uma pesquisa aplicada que resultou no presente artigo, estruturado em cinco partes, sendo a primeira esta introdução. A segunda parte constitui a fundamentação teórica sobre a gestão de processos, de forma geral e no serviço público, assim como elucida acerca do sistema ora pesquisado e como se deu sua implantação na instituição. Na terceira está descrita a metodologia para aplicação e análise da pesquisa, seguindo para a quarta parte, na qual constam os resultados e discussões acerca do questionário aplicado. Por fim, apresentam-se as conclusões e sugestões para futuras pesquisas.

\section{REVISÃO TEÓRICA}

No presente tópico serão apresentados os temas de estudo pertinentes ao trabalho. Inicialmente 
trata-se da gestão de processos de maneira mais ampla e generalizada, passando para a gestão de processos no serviço público. Por fim, é feita uma explanação sobre o Sistema Eletrônico de Informações - SEl, que é a ferramenta de gestão de processos em foco no presente estudo.

\section{Gestão de processos}

O processo pode ser compreendido como um modo de se realizar determinada tarefa de maneira mais organizada, evitando desvios durante a execução da atividade e o consequente retrabalho. O processo permite, a quem o realizará, ter conhecimento sobre o que será preciso para o seu início, possibilitando a definição prévia dos resultados que deverão ser atingidos. Assim, o processo pode ser definido como a introdução de insumos, chamada de entradas, em um ambiente formado por procedimentos, normas e regras que, ao processarem tais insumos, os transformam em resultados que serão enviados aos clientes do processo, caracterizando a saída (KIPPER et al., 2011).

Biazzi et al. (2011) corroboram com o entendimento ao afirmar que quando uma organização adota uma visão por processos, enfatiza-se a maneira como o trabalho é realizado. Dessa forma, segundo os autores, um processo é uma ordenação específica das atividades de trabalho no tempo e no espaço, com um começo, um fim e entradas e saídas claramente identificadas. Na mesma linha, Davenport (1993) entende que processo é o conjunto estruturado de atividades projetadas para produzir uma ênfase especificada em como o trabalho é realizado dentro de uma organização.

Nesse contexto, surge a gestão de processos, ou gestão por processos, que é uma metodologia para avaliação contínua, análise e melhoria do desempenho dos processos, principalmente daqueles que são mais impactantes na satisfação dos clientes e stakeholders em geral. É marcada por um grande envolvimento de todos dentro da organização, o que pode resultar em uma melhor satisfação dessas pessoas no trabalho, agilidade no andamento das atividades, desenvolvimento de habilidades, bem como no aumento da autoridade e autonomia individual (ROTONDARO, 2005, citado por SALGADO et al., 2013).

De acordo com Paim et al. (2009), todas as organizações, sem exceção, precisam coordenar o trabalho, e esses mecanismos de coordenação se relacionam intrinsecamente à forma como os recursos e as atividades estão projetados, ao modo como tais atividades são geridas no dia a dia e os meios pelos quais a organização irá gerar o aprendizado e promover as melhorias nas operações e na forma de coordenação do trabalho em si. Para os autores, a melhoria dos processos é uma ação básica para as organizações responderem às mudanças que ocorrem constantemente em seu ambiente de atuação. As organizações públicas não fogem desse cenário, sendo, portanto, de extrema importância a aplicação da gestão de processos no setor público.

\section{Gestão de processos no setor público}

As instituições públicas brasileiras têm buscado alternativas de gestão orientadas a resultados para o cidadão e com foco na qualidade dos processos internos, tendo em vista que um dos maiores desafios da administração pública brasileira ainda é de natureza gerencial (FALCÃO JUNIOR et al., 2016). 
Levando em conta, de modo específico, as Instituições Federais de Ensino Superior (IFES) e as imposições burocráticas às quais estão vinculadas, a maioria passa por dificuldades constantes, o que demanda um modelo de gestão facilitador de processos organizacionais, que as tornem mais ágeis e possibilite o acompanhamento do ritmo das mudanças sociais (LORENA, 2015).

Do ponto de vista de Ramos et al. (2019), utilizar a gestão por processos "permite o conhecimento dos procedimentos de execução dos processos, o que contribui para o alcance dos objetivos organizacionais por meio da melhoria na prestação dos serviços com qualidade, em menor tempo e com foco no cliente (interno e externo)".

Neste sentido, de acordo com o Guia de Gestão de Processos de Governo (BRASIL, 2011), observando que a tendência é que os processos sejam tratados como complexas e grandes redes organizacionais que se constituem para o alcance de resultados comuns, aumenta a necessidade de tecnologias que possibilitem o compartilhamento do conhecimento e a rápida tomada de decisão, o que torna indispensável o suporte ferramental no âmbito de processos, com destaque para os sistemas de automatização da gestão de processos no serviço público.

Ainda nesta direção, Araújo (2018) argumenta que a utilização de sistemas de informação está cada vez mais frequente na maioria das organizações, e que o processo de adoção desses sistemas leva em conta, cada vez mais, a constatação dos benefícios que envolvem os investimentos em tecnologias.

Neste contexto, a instituição em análise optou por realizar a automatização dos seus processos administrativos através da implantação de um sistema já utilizado por outros órgãos, o SEI - Sistema Eletrônico de Informações, que será tema do próximo tópico do presente artigo.

\section{O Sistema Eletrônico de Informações - SEI}

Com o intuito de melhoria e fluidez na tramitação dos processos, sem mencionar a colaboração com a sustentabilidade e diminuição de custos na Administração Pública, foi publicado o Decreto no 8.539, de 08 de outubro de 2015, que instituiu o uso do meio eletrônico para a realização do processo administrativo no âmbito dos órgãos e das entidades da administração pública federal direta, autárquica e fundacional.

No âmbito do Ministério da Educação, mais especificamente na Universidade Tecnológica Federal do Paraná, foi instituído, em 31 de julho de 2017, o uso do Sistema Eletrônico de Informações (SEI), software livre desenvolvido pelo Tribunal Regional Federal da 4a Região (TRF4), o qual foi escolhido como solução de processo eletrônico para o projeto Processo Eletrônico Nacional (PEN), iniciativa conjunta de órgãos e entidades de diversas esferas da administração pública, com o objetivo de construir uma infraestrutura pública de processos e documentos administrativos eletrônicos (BRASIL, 2019).

Uma das principais vantagens do sistema é o controle de nível de acesso, ou seja, é possível gerenciar a criação e tramitação de processos e documentos com informações sensíveis, conferindo acesso somente às unidades ou a usuários específicos. Outra vantagem é a tramitação do processo em múltiplas unidades, as quais podem ser demandadas, tomar providências e manifestar-se simultaneamente.

Além disso, dispõe, ainda, de ferramentas para controle de prazos e tempo de tramitação do 
processo, base de conhecimento, estatísticas da unidade, pesquisa em todo teor, acompanhamento especial, criação de modelos de documentos e textos padrão, organização de processos em blocos e disponibilização para assinaturas simultâneas, entre outros comandos.

Na UTFPR, o Escritório de Processos (EPROC) e o Comitê Gestor do SEI são os responsáveis pela administração do Sistema Eletrônico de Informações. Há, também, o Grupo de Suporte ao Usuário (GSU), constituído por servidores designados por portaria, que atuaram como facilitadores durante a implantação do sistema e atualmente prestam suporte aos usuários do SEI em todos os câmpus da UTFPR.

O EPROC, em conjunto com os facilitadores das áreas e os patrocinadores dos processos, define as bases de conhecimento, os tipos de processos, documentos, modelos, entre outros. Além disso, o EPROC eventualmente desenvolve módulos que são integrados ao SEI e fornecem informações à área de tecnologia da informação sobre webservices disponíveis para integração do SEI com os sistemas corporativos da universidade.

Diante do exposto, tendo em vista as significativas mudanças decorrentes da implantação do SEI na UTFPR, e considerando que a área de gestão de pessoas foi utilizada como piloto para tal implantação, evidencia-se a relevância de analisar como ocorreu essa transição de procedimentos, e se, de fato, na opinião dos servidores da área, houve uma melhoria no desenvolvimento e gerenciamento das atividades de trabalho, uma vez que tais setores possuem um número expressivo de processos de trabalho.

\section{METODOLOGIA}

Este artigo caracteriza-se como uma pesquisa descritiva, a qual buscou analisar a percepção dos servidores lotados na Diretoria de Gestão de Pessoas e nas Coordenadorias de Gestão de Recursos Humanos da UTFPR sobre a implantação do SEl, e como ele afetou o desenvolvimento do trabalho nestes setores.

Gil (2008) menciona que as pesquisas descritivas:

Têm como objetivo primordial a descrição das características de determinada população ou fenômeno ou o estabelecimento de relações entre variáveis. São inúmeros os estudos que podem ser classificados sob este título e uma de suas características mais significativas está na utilização de técnicas padronizadas de coleta de dados.

Para tanto, foi elaborado um questionário no modelo estruturado, dividido em duas partes, sendo a primeira referente a questões sociodemográficas e funcionais que compreenderam o levantamento de: idade, sexo, tempo de trabalho na UTFPR e no setor, última formação concluída, cargo e câmpus de lotação. A segunda parte abordou os temas relacionados ao sistema estudado, referentes à capacitação para uso do mesmo, frequência de utilização do produto, bem como os quesitos de percepção voltados ao desenvolvimento do trabalho com a utilização dessa nova ferramenta.

O questionário foi composto, ao todo, por 31 questões, e aplicado por meio de formulário eletrônico, tendo em vista que seu público-alvo se encontrava nas 13 cidades onde a Universidade possui Câmpus, ou seja, Apucarana, Campo Mourão, Cornélio Procópio, Curitiba, Dois Vizinhos, Francisco Beltrão, Guarapuava, Londrina, Medianeira, Pato Branco, Ponta Grossa, Santa Helena e Toledo. Previamente à aplicação da pesquisa, foi solicitada a anuência da dirigente máxima do setor de gestão de pessoas da universidade, sendo 
autorizada a sua realização.

Quanto às questões relativas à percepção dos servidores sobre o sistema, foram apresentadas 22 afirmativas com opções de resposta determinadas por uma escala do tipo Likert de cinco pontos, que consiste, segundo Gil (2008), na solicitação para um "certo número de pessoas que manifestem sua concordância ou discordância em relação a cada um dos enunciados, segundo uma graduação". Algumas afirmativas foram extraídas ou adaptadas da pesquisa de Pires-Fulton et al. (2018), e outras foram elaboradas pelos autores do presente estudo.

Por último, os dados foram tratados mediante condensação e análise das informações, culminando na sua interpretação, na elaboração das conclusões e sugestões para estudos futuros.

\section{RESULTADOS E DISCUSSÃO}

O universo pesquisado foi composto de 88 servidores lotados na Diretoria de Gestão de Pessoas (DIRGEP), na Reitoria, ou nas Coordenadorias de Gestão de Recursos Humanos (COGERHs) dos 13 câmpus da UTFPR. Foram obtidas 45 respostas, o que corresponde a $51,1 \%$ do universo pesquisado, conforme evidenciado na Tabela 1.

Tabela 1: Participação dos servidores da DIRGEP e COGERHs na pesquisa.

\begin{tabular}{llll}
\hline Variável & Descrição & Frequência Absoluta & Frequência Relativa \% \\
\hline Câmpus/Unidade & Apucarana & 1 & 2,2 \\
& Campo Mourão & 2 & 4,4 \\
& Cornélio Procópio & 2 & 4,4 \\
Curitiba & 6 & 13,3 \\
Dois Vizinhos & 2 & 4,4 \\
Francisco Beltrão & 1 & 2,2 \\
Guarapuava & 2 & 4,4 \\
Londrina & 3 & 6,7 \\
Medianeira & 4 & 8,9 \\
& Pato Branco & 3 & 6,7 \\
Ponta Grossa & 4 & 8,9 \\
Reitoria & 12 & $\mathbf{2 6 , 7}$ \\
Santa Helena & 2 & 4,4 \\
Toledo & 1 & 2,2 \\
\hline Total & 45 & 100,0 \\
\hline
\end{tabular}

Fonte: adaptado de Stacciarini (2010).

\section{Características sociodemográficas e funcionais dos servidores}

O levantamento sociodemográfico e funcional dos servidores foi constituído por idade, sexo, tempo de trabalho na UTFPR e no setor, última formação concluída, cargo e câmpus de lotação. Observa-se a predominância de gênero do sexo feminino, com 71,1\%, equivalente à participação de 32 mulheres e, consequentemente, 13 homens.

Quanto à idade, com o intuito facilitar a análise, optou-se por estabelecer conjuntos de faixas etárias: i) entre 18 e 30 anos; ii) entre 31 e 45 anos; iii) entre 46 e 55 anos; e iv) 56 anos ou mais. Sendo predominante a faixa etária entre 31 e 45 anos, com 31 pessoas, o que corresponde a 68,9\% da amostra, e a 24 mulheres e 7 homens.

O tempo de trabalho na UTFPR e no setor de gestão pessoas, também foram escalonados por faixas: 
i) até 1 ano; ii) de 1 a 5 anos; iii) de 6 a 10 anos; e iv) 11 anos ou mais. As faixas 'de 1 a 5 anos' e 'de 6 a 10 anos' tiveram 16 respondentes cada uma, correspondendo a 35,6\% cada, para o tempo de trabalho na universidade. Já no setor de gestão pessoas $46,7 \%$ estão de 1 a 5 anos trabalhando nesta área. Encontramse na faixa de 'até 1 ano', 2 pessoas, tanto de tempo de UTFPR quanto no setor de gestão de pessoas. Dos 11 entrevistados que estão há 11 anos ou mais na instituição, 9 também estão pelo mesmo período na área de gestão de pessoas, e dentre estes 9, 7 têm idade entre 46 e 55 anos e 2 entre 31 e 45 anos, sendo 7 mulheres e 2 homens. A Tabela 2 elucida as características sociodemográficas e funcionais dos servidores pesquisados.

Tabela 2: Características sociodemográficas e funcionais dos servidores.

\begin{tabular}{llll}
\hline Variável & Descrição & Frequência Absoluta & Frequência Relativa \% \\
\hline Idade & Entre 18 e 30 anos & 6 & 13,3 \\
& Entre 31 e 45 anos & 31 & $\mathbf{6 8 , 9}$ \\
& Entre 46 e 55 anos & 7 & 15,6 \\
& 56 anos ou mais & 1 & 2,2 \\
& Total & 45 & 100,0 \\
\hline Sexo & Feminino & 32 & $\mathbf{7 1 , 1}$ \\
& Masculino & 13 & 28,9 \\
& Total & 45 & 100,0 \\
\hline Tempo de trabalho & Até 1 ano & 2 & 4,4 \\
na UTFPR & De 1 a 5 anos & 16 & $\mathbf{3 5 , 6}$ \\
& De 6 a 10 anos & 16 & $\mathbf{3 5 , 6}$ \\
& 11 anos ou mais & 11 & 24,4 \\
& Total & 45 & 100,0 \\
\hline Tempo de trabalho & Até 1 ano & 2 & 4,4 \\
na área de gestão & De 1 a 5 anos & 21 & $\mathbf{4 6 , 7}$ \\
de pessoas & De 6 a 10 anos & 13 & 28,9 \\
& 11 anos ou mais & 9 & 20,0 \\
& Total & 45 & 100,0 \\
\hline
\end{tabular}

Fonte: adaptado de Stacciarini (2010).

Na variável 'Última formação concluída' foram considerados os seguintes graus de escolaridade: i) Ensino Médio; ii) Graduação; iii) Especialização; iv) Mestrado; v) Doutorado; vi) Pós-doutorado. A maioria dos servidores (51,1\%) possui o título de especialista, 31,1\% têm mestrado, 13,3\% graduação e 4,4\% doutorado, como última formação. Não há servidores, entre os respondentes, com apenas ensino médio, ou que já tenham realizado pós-doutorado.

Quanto à questão "Cargo ocupado", observou-se que houve confusão por parte dos pesquisados em relação ao conceito, pois 5 deles responderam com as funções que ocupam na instituição. Cabe esclarecer que cargos públicos são "as mais simples e indivisíveis unidades de competência a serem expressadas por um agente, previstas em número certo, com denominação própria, retribuídas por pessoas jurídicas de Direito Público e criadas por lei" e que as funções públicas são "plexos unitários de atribuições, criados por lei, correspondentes a encargos de direção, chefia ou assessoramento, a serem exercidas por titular de cargo efetivo, da confiança da autoridade que as preenche" (MELLO, 2013).

Apesar da situação acima relatada, foi possível verificar que 40 pessoas responderam corretamente à questão, e dentre estes, 29 ocupam o cargo de Assistente em Administração, cujo nível de escolaridade exigido para ingresso é o ensino médio, e possuem como última titulação Graduação (5), Especialização (16) ou Mestrado (8). Ou seja, embora a maioria dos respondentes ocupe cargo de nível médio, essa maioria 
possui, na sua integralidade, no mínimo o título de graduação, ao menos $55 \%$ possui especialização, e quase $30 \%$ possui mestrado. Os demais cargos ocupados citados na pesquisa foram: Administrador (3), Psicólogo (2), Assistente Social (2), Auxiliar em Administração (2), Técnico em Contabilidade (1), e Técnico em Assuntos Educacionais (1).

\section{Percepção dos servidores sobre o SEI}

A segunda parte da pesquisa referiu-se mais objetivamente à implantação do Sistema Eletrônico de Informações na área de gestão de pessoas, com questões relativas à capacitação para usabilidade da ferramenta, à frequência de utilização do sistema e os quesitos de percepção dos usuários, estes últimos estão evidenciados no quadro 1.

Quadro 1: Questões relativas.

1. O SEI mudou significativamente a forma de realizar o meu trabalho.

2. As mudanças trazidas pelo SEI foram positivas.

3. Tive dificuldades para utilizar o SEI.

4. Com o SEI, senti agilidade na tramitação dos processos.

5. Com o SEl, percebi a diminuição de gargalos.

6. O SEI trouxe benefícios para a área de gestão de pessoas.

7. O SEl é de fácil utilização.

8. O SEI apresenta uma interface intuitiva.

9. O uso do SEI melhorou a qualidade do meu trabalho.

10. O uso do SEl aumentou a minha produtividade.

11. O uso do SEI permitiu realizar minhas tarefas mais rapidamente.

12. O SEI me dá um controle maior sobre o meu trabalho.

13. No geral, acho que o uso do SEl é vantajoso para o meu trabalho.

14. O SEl facilitou a realização dos processos da área de cadastro.

15. O SEI facilitou a realização dos processos da área de pagamento.

16. O SEI facilitou a realização dos processos da área de benefícios.

17. O SEI facilitou a realização dos processos da área de desenvolvimento/capacitação.

18. O SEI facilitou a realização dos processos da área de movimentação de pessoas.

19. O SEI facilitou a realização dos processos da área de estágio.

20. Os processos que realizo no SEI possuem base de conhecimento.

21. As bases de conhecimento fornecem orientações suficientes para a correta execução dos processos.

22. Prefiro a tramitação de processos via SEI à tramitação física.

Conforme se demonstra na Tabela 3, a maioria dos entrevistados fez algum curso de capacitação para utilizar o SEI $(84,4 \%)$, sendo que 24 servidores realizaram tanto o curso ofertado pela própria UTFPR quanto o curso 'Sistema Eletrônico de Informações - SEI! USAR', promovido pela Escola Nacional de Administração Pública (Enap). Houve 7 participantes que não realizaram nenhum curso de capacitação. Embora o número de servidores capacitados seja elevado, outras respostas evidenciaram certo nível de dificuldade na utilização do sistema.

Dentre as pessoas que realizaram os dois cursos de capacitação, todas utilizam o SEI muito/quase sempre. Dentre a amostra completa, 2 pessoas utilizam consideravelmente/frequentemente o sistema, 1 pouco/raramente, e 42 utilizam muito/quase sempre. Ou seja, todos os participantes utilizam o SEI.

As respostas para os quesitos de percepção foram baseadas na escala Likert de cinco pontos, sendo: 1: concorda muito; 2: concorda um pouco; 3: indeciso; 4: discorda um pouco; 5: discorda muito.

Dos 45 respondentes, 40 concordam muito e 4 concordam um pouco que o SEl mudou significativamente a forma de realizar o seu trabalho, ou seja, a extrema maioria de $97,8 \%$ concorda, de 
alguma forma, com essa afirmativa. A grande maioria também concorda, muito ou pouco, que as mudanças trazidas pelo SEI foram positivas, correspondendo a 93,3\% das respostas.

Tabela 3: Capacitação e frequência de utilização do SEI.

\begin{tabular}{|c|c|c|c|}
\hline Variável & Descrição & $\begin{array}{l}\text { Frequência } \\
\text { Absoluta }\end{array}$ & $\begin{array}{l}\text { Frequência } \\
\text { Relativa \% }\end{array}$ \\
\hline Fez algum curso de & Sim & 38 & 84,4 \\
\hline capacitação para utilizar o & Não & 7 & 15,6 \\
\hline SEI? & Total & 45 & 100,0 \\
\hline \multirow[t]{7}{*}{ Se sim, qual(is)? } & $\begin{array}{l}\text { Sistema Eletrônico de Informações - SEI! USAR - Promovido pela } \\
\text { Escola Nacional de Administração Pública - Enap }\end{array}$ & 8 & 17,8 \\
\hline & Curso promovido pela UTFPR & 6 & 13,3 \\
\hline & Outro & 0 & 0,0 \\
\hline & Sistema Eletrônico de Informações - SEI! USAR - Promovido pela & & \\
\hline & $\begin{array}{l}\text { Escola Nacional de Administração Pública - Enap / Curso promovido } \\
\text { pela UTFPR }\end{array}$ & 24 & 53,3 \\
\hline & Não realizaram nenhum curso & 7 & 15,6 \\
\hline & Total & 45 & 100,0 \\
\hline \multirow{5}{*}{$\begin{array}{l}\text { Com que frequência utiliza } \\
\text { o SEI nas suas rotinas de } \\
\text { trabalho? }\end{array}$} & Não utiliza & 0 & 0,0 \\
\hline & Utiliza pouco/raramente & 1 & 2,2 \\
\hline & Utiliza consideravelmente/frequentemente & 2 & 4,4 \\
\hline & Utiliza muito/quase sempre & 42 & 93,3 \\
\hline & Total & 45 & 100,0 \\
\hline
\end{tabular}

Fonte: adaptado de Stacciarini (2010).

Quanto às dificuldades para utilização do sistema, $44,4 \%$ concordaram muito ou pouco com essa afirmativa. Dentre esses, $70 \%$ possui mais de 05 anos de trabalho na instituição, $40 \%$ possui até 05 anos trabalhados na área de gestão de pessoas, e $80 \%$ já realizaram algum curso de capacitação para utilização do sistema. Em contraponto, ao analisar as respostas do item $7,82,2 \%$ concordaram que o SEl é de fácil utilização.

Dois dos fatores que apresentaram menores índices de muita concordância, equivalente a apenas 20\%, são o 8 'O SEl apresenta uma interface intuitiva”, e o 20 'Os processos que realizo no SEI possuem base de conhecimento'. O fator 8 também foi o que apresentou maior índice de pouca discordância, com 17,8\%. $E$, ainda com relação às bases de conhecimento, $51,1 \%$ concordam um pouco que elas fornecem orientações suficientes para a correta execução dos processos e $26,7 \%$ concordam muito.

Entre os respondentes, $71,1 \%$ concordam, muito ou pouco, que o SEI possibilitou a diminuição de gargalos antes existentes nos processos de trabalho. $86,7 \%$ concordam, muito ou pouco, que, com o SEI, sentiram agilidade na tramitação dos processos, e 93,3\% também concordam que o SEI aumentou a sua produtividade. Ainda, $91,1 \%$ dos respondentes concordam que o SEI permitiu que suas tarefas sejam realizadas com maior rapidez, e que ele melhorou a qualidade do seu trabalho.

Com relação aos itens 14 a 19, que afirmam que o SEI facilitou a realização dos processos das principais áreas dos setores de gestão de pessoas e recursos humanos, que são cadastro, pagamento, benefícios, desenvolvimento/capacitação, movimentação de pessoas e estágio, o item que apresenta maior nível de discordância refere-se à área de estágio, que é também o que apresenta menor nível de concordância, com 13,3\% e 64,5\%, respectivamente. Este item também foi o que atingiu o maior número de respostas 'indeciso', equivalente e $22,2 \%$.

A segunda área com maior nível de discordância foi a de benefícios, com um índice de 8,9\%. A área 
de pagamento foi a segunda com menor nível de concordância, com 68,9\%. Todas as demais áreas apresentaram nível de concordância superior a 70\%, com ênfase para a área de movimentação de pessoas, cujo nível de concordância foi de 77,8\%, e que também apresentou o menor índice de respostas 'indeciso', 15,6\%. Analisando a afirmativa 12 , 'O SEI me dá um controle maior sobre o meu trabalho', 8,9\% dos respondentes discordam um pouco, $66,7 \%$ concordam muito, $20 \%$ concordam pouco, e $4,4 \%$ ficaram indecisos na resposta.

Por fim, o item 22, 'Prefiro a tramitação de processos via SEl à tramitação física', foi o que atingiu o segundo maior índice de muita concordância, correspondente a $86,7 \%$ dos respondentes, seguido dos itens 'As mudanças trazidas pelo SEI foram positivas', 'O SEI trouxe benefícios para a área de gestão de pessoas', 'No geral, acho que o uso do SEI é vantajoso para o meu trabalho', com $77,8 \%$ de muita concordância cada, e, respectivamente, índices de pouca concordância de 15,6\%, 13,3\% e 7,8\%, que também são bastante relevantes.

\section{CONCLUSÕES}

Através da pesquisa realizada, é possível afirmar que a maioria dos servidores da área de gestão de pessoas aprova/apoia a utilização do Sistema Eletrônico de Informações. Fica evidenciado que, apesar de apresentar significativas mudanças na forma de trabalho e nas rotinas da área, a implantação do SEI e a consequente informatização dos processos foi benéfica.

A alteração na forma de tramitação dos processos, do meio físico ao digital, impôs significativas mudanças para a área, contudo, considerando que a pesquisa foi realizada após aproximadamente dois anos e meio da implantação do sistema, foi possível perceber que os usuários defendem que em todas as principais áreas de gestão de pessoas a implantação do sistema proporcionou melhoria nos processos.

Geralmente mudanças mais profundas não são bem vistas. Quando se planeja implementar um novo processo de trabalho, que causará grande impacto na organização/instituição, pode haver um forte receio e até mesmo descrédito por parte dos agentes envolvidos quanto aos seus benefícios. Esse resultado mostra que, apesar de certo nível de desconforto/transtorno que tais processos representam, se a mudança for adequadamente estudada e planejada, há mais chances de se conseguir um bom resultado em sua implantação e uma boa aceitação por parte dos usuários.

Também foi possível constatar que a utilização do SEI possibilitou maior controle dos processos realizados, e, para a grande maioria dos respondentes, proporcionou um aumento na produtividade e mais celeridade na realização do trabalho. Um sistema eletrônico, que permite a tramitação digital do processo, bem como o acompanhamento em tempo real de seu status, possibilitou maior controle do trabalho aos servidores da área de gestão de pessoas, o que também pode representar maior segurança na execução do trabalho e na rastreabilidade do processo. A informatização também possibilita maior rapidez na realização das atividades, tendo em vista que permite assinaturas digitais de documentos, tramitação instantânea entre câmpus, entre outros.

A não inclusão de questões discursivas impossibilitou a revelação de possíveis pontos não levantados 
pelos pesquisadores, bem como impossibilitou uma análise qualitativa e mais detalhada com relação ao evento investigado. Assim, sugere-se que pesquisas futuras possam realizar esse tipo de abordagem, visando complementar e aprimorar o presente estudo. Sugere-se, ainda, a aplicação de pesquisa similar para os usuários dos serviços da área de Gestão de Pessoas e Recursos Humanos, que são os demais servidores da instituição, a fim de analisar o seu ponto de vista e verificar se a implantação do SEI nessa área também foi benéfica aos usuários do setor.

\section{REFERÊNCIAS}

ARAÚJO, J. M.. Inovação de processos: implementação do Sistema Eletrônico de Informações na Universidade de Brasília. 2018. Dissertação (Mestrado em Gestão Pública) Universidade de Brasília, Brasília, 2018.

BIAZZI, M. R.; MUSCAT, A. R. N.; BIAZZI, J. L.. Modelo de aperfeiçoamento de processos em instituições públicas de ensino superior. Revista Gestão \& Produção. São Carlos, v.18, n.4, p.869-880, 2011. DOI: https://doi.org/10.1590/S0104-530X2011000400013

BRASIL. Decreto n.8539 de 08 de outubro de 2015. Dispõe sobre o uso do meio eletrônico para a realização do processo administrativo no âmbito dos órgãos e das entidades da administração pública federal direta, autárquica e fundacional. Brasília: DOU, 2015.

BRASIL. Guia de Gestão de Processos de Governo. Brasília: MPOG, 2011.

BRASIL. Portal do Processo Eletrônico Nacional. Brasília: ME, 2019.

DAVENPORT, T. H.. Process Innovation: Reengineering work through information technology. Massachusetts: Harvard Business School Press, 1993.

DOMINGUES, F. M. F. V.; XAVIER, W. G.; BIROCHI, R.. Gestão por processos: uma análise da ferramenta de gestão utilizada no Poder Judiciário de Santa Catarina. Revista Eletrônica de Estratégia \& Negócios, Florianópolis, v.8, n.1, p.199-238, 2015. DOI:

http://dx.doi.org/10.19177/reen.v8e12015199-238

FALCÃO JUNIOR, M. A. G.; SANTOS, R. N. M.. A gestão de processos na análise das atividades de seleções públicas simplificadas: estudo de caso em uma prefeitura. Navus: Revista de Gestão e Tecnologia, Florianópolis, v.6, n.2, p.619, 2016. DOI:

https://doi.org/10.22279/navus.2016.v6n2.p06-19.324

GIL, A. C.. Métodos e técnicas de pesquisa social. 6 ed. São Paulo: Atlas, 2008.

KIPPER, L. M; ELLWANGER, M. C.; JACOBS, G.; NARA, E. O. B. FROZZA, R.. Gestão por processos: comparação e análise entre metodologias para implantação da gestão orientada a processos e seus principais conceitos. Tecno-Lógica, Santa Cruz do Sul, v.15, n.2, p.89-99, 2011.
LORENA, A. L. F.. A Contribuição Estratégica da Ouvidoria Pública para a Gestão de Processos de Negócios nas IFES. In: COLÓQUIO INTERNACIONAL DE GESTÃO UNIVERSITÁRIA, 15. Anais. Mar del Plata, 2015.

MELLO, C. A. B.. Curso de Direito Administrativo. 30 ed. Rio de Janeiro: Malheiros, 2013.

PAIM, R.; CARDOSO, V.; CAULLIRAUX, H.; CLEMENTE, R. Gestão de Processos: pensar, agir e aprender. Rio de Janeiro: Bookman, 2009.

PEREIRA, L. F. A.; SILVA, S. S.. Burocracia no Setor de Recursos Humanos de uma Autarquia. RASI: Revista de Administração, Sociedade e Inovação, Volta Redonda, v.2, n.2, p.118-131, 2016. DOI: https://doi.org/10.20401/rasi.2.2.86

PIRES-FULTON, D.; FARIAS, J.; ALFINITO, S.; ALMEIDA, J. Adoção do Sistema Eletrônico de Informações (SEI) pelo Ministério da Justiça: uma avaliação na ótica de usuários. Revista do Serviço Público, v.69, n.4, p.1015-1036, 2018. DOI: https://doi.org/10.21874/rsp.v69i4.3624

RAMOS, K. H. C.; MONTEZANO, L.; COSTA JÚNIOR, R. L.; SILVA, A. C. A. M.. Dificuldades e benefícios da implantação da gestão de processos em organização pública federal sob a ótica dos servidores. Revista Gestão \& Tecnologia, Pedro Leopoldo, v.19, n.4, p.161-186, 2019. DOI: https://doi.org/10.20397/2177-6652/2019.v19i4.1593

SALGADO, C. C. R.; AIRES, R. F. F.; WALTER, F.; ARAÚJO, A. G.. Contribuições à melhoria de processos organizacionais: uma avaliação empírica sob a perspectiva de mapeamento de processos em uma unidade da Universidade Federal da Paraíba. Holos, v.1, p.151-168, 2013. DOI: https://doi.org/10.15628/holos.2013.1034

SILVA, V. N.; RISSI, M.. Gestão de projetos na gestão estratégica universitária: um estudo bibliométrico. Revista Capital Científico, v.17, n.4, p.44-64, 2019.

STACCIARINI, M. R.. Percepção de Suporte Organizacional: um estudo de caso na Secretaria de Recursos Humanos do Ministério do Planejamento, Orçamento e Gestão. In: CAMÕES, M. R. D. S.; PANJOTA, M. J.; BERGUE, S. T.. Gestão de pessoas: bases teóricas e experiências no setor público. Brasília: ENAP, 2010. p.283-300.

A CBPC - Companhia Brasileira de Produção Científica (CNPJ: 11.221.422/0001-03) detém os direitos materiais desta publicação. Os direitos referem-se à publicação do trabalho em qualquer parte do mundo, incluindo os direitos às renovações, expansões e disseminações da contribuição, bem como outros direitos subsidiários. Todos os trabalhos publicados eletronicamente poderão posteriormente ser publicados em coletâneas impressas sob coordenação da Sustenere Publishing, da Companhia Brasileira de Produção Científica e seus parceiros autorizados. Os (as) autores (as) preservam os direitos autorais, mas não têm permissão para a publicação da contribuição em outro meio, impresso ou digital, em português ou em tradução. 